\title{
Limits of photon-mediated interactions in one-dimensional photonic baths
}

\author{
Eduardo Sánchez-Burillo, ${ }^{1}$ Diego Porras, ${ }^{2}$ and Alejandro González-Tudela $\odot^{2, *}$ \\ ${ }^{1}$ Max-Planck-Institut für Quantenoptik, D-85748 Garching, Germany \\ ${ }^{2}$ Instituto de Física Fundamental IFF-CSIC, Calle Serrano 113b, E-28006 Madrid, Spain
}

(Received 23 March 2020; accepted 15 June 2020; published 8 July 2020)

\begin{abstract}
The exchange of off-resonant propagating photons between distant quantum emitters induces coherent interactions among them. The range of such interactions, and whether they are accompanied by dissipation, depends on the photonic energy dispersion, its dimensionality, and/or the light-matter couplings. We characterize the limits of photon-mediated interactions for the case of generic one-dimensional photonic baths under the typical assumptions, i.e., finite range hoppings for the photonic bath plus local and rotating-wave light-matter couplings. In that case, we show how, irrespective of the system's parameter, the coherent photon-mediated interactions can always be written as a finite sum of exponentials and thus cannot display a power-law asymptotic scaling. As an outlook, we show how by relaxing some of these conditions, e.g., going beyond local light-matter couplings (e.g., giant atoms) or with longer-range photon hopping models, power-law interactions can be obtained within certain distance windows or even in the asymptotic regime for the latter case.
\end{abstract}

DOI: 10.1103/PhysRevA.102.013709

\section{INTRODUCTION}

Even perfectly isolated, distant quantum emitters can interact through the fluctuations of the electromagnetic field (photons) around them [1,2]. The exchange of off-resonant photons, which are the ones with energies that are different from the emitter's transition frequency, leads to reversible excitation transfer between the emitters because photons are only virtually populated during the exchange process. In free space [1,2], for example, these interactions $\left(J_{i j}\right)$ decay with the distance between emitters $\left(r_{i j}\right)$ as a power law $r_{i j}^{-3(1)}$ in the near (far) field. Such power-law coherent interactions have raised a lot of interest because they can be harnessed for quantum information $[3,4]$ or simulation tasks, e.g., to explore long-range interacting spin models that are known to lead to many unconventional phenomena [5-24]. Unfortunately, in free space, these dipolar interactions are accompanied by collective (and individual) dissipative couplings $\left(\gamma_{i j}\right)$ induced by the resonant photons, precluding many of their potential applications.

A way of avoiding this problem consists in modifying the photonic environment around the emitters to inhibit the modes around the emitters' transition frequencies [25]. This can be done, for example, in photonic crystals [26-29], where one can indeed cancel the associated dissipation, i.e., $\gamma_{i j}=0$, by tuning the emitter's frequency into a photonic band-gap region. This cancellation generally comes at the price, however, of an exponential localization of the interactions, i.e., $J_{i j} \propto$ $e^{-r_{i j} / \xi}$, whose characteristic length $\xi$ can be tuned by changing the band energy dispersion and the emitter's detuning to the band edge [30,31]. The only exceptions to this exponential localization of such photon-mediated interactions, to our knowledge, have been found in high-dimensional singular

\footnotetext{
*a.gonzalez.tudela@csic.es
}

band gaps [32-36], where power-law $J_{i j}$ interactions have been predicted with no associated dissipation. While some attempts with similar energy dispersions have been explored in one dimension (1D) [37], the emergence of such power-law coherent interactions mediated by one-dimensional photonic environments remains, so far, elusive.

Motivated by this quest, in this manuscript we study the limits in the range of photon-mediated interactions induced by one-dimensional environments. Given the variety of experimental platforms available nowadays to explore such quantum optical effects, ranging from photonic crystals [38,39], circuit QED metamaterials [40,41], and subwavelength atomic arrays $[42,43]$, to state-dependent optical lattices $[44,45]$, we provide results for generic one-dimensional models using a minimal set of assumptions: local and excitation-conserving light-matter couplings, together with finite-range hoppings for the bath. With these assumptions, we are able to show that the quantum emitters' interactions can always be written as a finite sum of exponential terms and can thus never display a power-law decay, irrespective of the model considered. In addition, we study situations where some of these assumptions are broken, and show how one could obtain (quasi)-power-law interactions. For example, we show that nonlocal light-matter couplings, such as the ones enabled by giant atoms [46,47], open up the possibility of mimicking power-law interactions up to certain distances in a controlled way. Furthermore, we also study baths with longer-range hoppings, which can lead to power-law interactions even in the asymptotic limit.

The manuscript is structured as follows: In Sec. II, we first write the generic light-matter Hamiltonian that we will consider throughout the manuscript. Then, we derive the effective photon-mediated interactions in Sec. III. Afterwards, in Sec. IV, we explore the possibilities of obtaining powerlaw interactions by breaking some of the assumptions of the general model considered in Sec. II. Finally, we summarize our findings in Sec. V. 


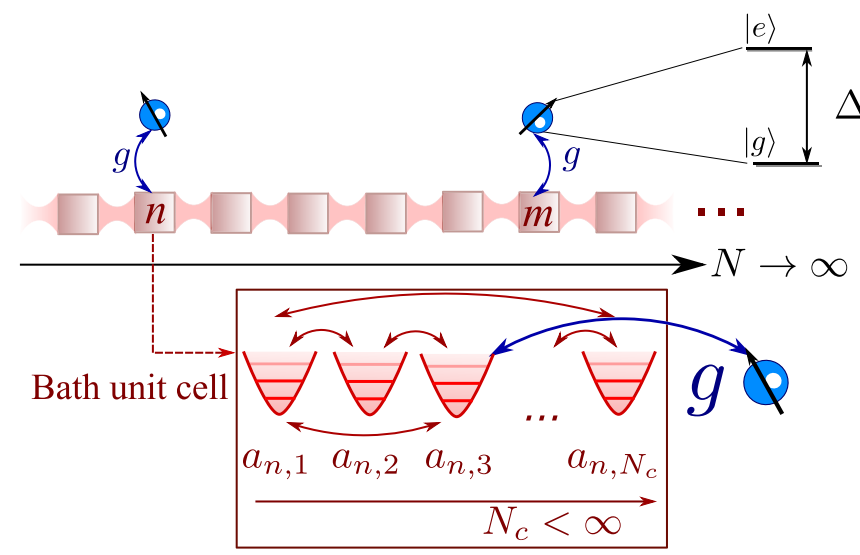

FIG. 1. Scheme of model considered: The photonic bath is described by a set of $N$ unit cells (that we will consider to be infinite) composed of $N_{c}$ (finite) coupled resonator modes. We use bosonic operators $a_{n, \alpha}^{\dagger}$ to describe the $\alpha$ resonator mode of the $n$th unit cell. We consider the energy of the discrete resonators $a_{n, \alpha}$ to be equal, and take it as the energy reference of the problem. Two-level $(|g\rangle,|e\rangle)$ quantum emitters, with detuning $\Delta$ from the energy of the resonators, are locally coupled with strength $g$ to a particular resonator of a given unit cell $n$.

\section{MODEL}

Here we introduce the different terms of the Hamiltonian of the general model that we will consider throughout the manuscript (see Fig. 1). The photonic bath is described by $N$ unit cells with $N_{c}$ discrete coupled resonators each, that is, a one-dimensional model with $N_{c}$ sublattices. We only allow for hoppings within the same unit cell or between nearestneighbor unit cells. Thus, we can capture any one-dimensional photonic bath with finite-range hoppings up to $N_{c}$ neighbors. We use bosonic operators $a_{n, \alpha}, a_{n, \alpha}^{\dagger}$ to describe the photonic excitations of the $\alpha$ th resonator within the $n$th unit cell of the lattice. As we are interested in the limit $N \rightarrow \infty$, we can safely take periodic boundary conditions and write the bath Hamiltonian in the following form:

$$
H_{B}=\sum_{k}\left(\tilde{a}_{k, 1}^{\dagger} \ldots \hat{a}_{k, N_{c}}^{\dagger}\right) h_{B}(k)\left(\begin{array}{c}
\hat{a}_{k, 1} \\
\vdots \\
\hat{a}_{k, N_{c}}
\end{array}\right),
$$

where we use the $\hat{.}$ notation to distinguish the bosonic operators defined in momentum space as $\hat{a}_{k, \alpha}=$ $\frac{1}{\sqrt{N}} \sum_{n=1}^{N} e^{-i k n} a_{n, \alpha}$, with $\alpha=1, \ldots, N_{c}$ [48]. The matrix $h_{B}(k)$ is a Hermitian matrix, which can be written with full generality as

$$
h_{B}(k)=\left(\begin{array}{ccccc}
\delta_{1}(k) & f_{12}(k) & f_{13}(k) & \ldots & f_{1 N}(k) \\
f_{12}^{*}(k) & \delta_{2}(k) & f_{23}(k) & \ldots & f_{2 N}(k) \\
f_{13}^{*}(k) & f_{23}^{*}(k) & \delta_{3}(k) & \ldots & f_{3 N}(k) \\
\ldots & \ldots & \ldots & \ldots & \ldots \\
f_{1 N}^{*}(k) & f_{2 N}^{*}(k) & f_{3 N}^{*}(k) & \ldots & \delta_{N}(k)
\end{array}\right),
$$

where $\delta_{\alpha}(k)$ is the dispersion relation of the $\alpha$ th sublattice and $f_{\alpha, \beta}(k)$ is the function characterizing the interactions between the $\alpha$ th and $\beta$ th sublattices. This bath Hamiltonian can be diagonalized, resulting in $N_{c}$ energy bands $\omega_{\alpha}(k)$.
The $N_{e}$ quantum emitters are described as two-level systems $(|g\rangle,|e\rangle)$, with energies $\Delta$ (see Fig. 1). Thus, their internal dynamics is simply given by

$$
H_{S}=\Delta \sum_{j=1}^{N_{e}} \sigma_{j}^{\dagger} \sigma_{j}
$$

with $\sigma_{j}=|g\rangle_{j}\langle e|$ being the spin-operator transition of the $j$ th atom. As mentioned in Sec. I, we consider that these emitters are locally coupled to the environment (local-dipole approximation). This implies that each quantum emitter couples only to one of the resonators of a given unit cell of the lattice. In addition, we describe the light-matter interaction through a Jaynes-Cummings Hamiltonian (rotating-wave regime approximation). The latter is a good description of light-matter interaction as long as the coupling strength is much smaller than the emitter and bath frequencies [49], as is the typical case in most of the systems of interest to us. Under these assumptions, the light-matter Hamiltonian reads

$$
H_{\mathrm{int}}=\sum_{j=1}^{N_{e}}\left(g \sigma_{j}^{\dagger} a_{n_{j}, \alpha_{j}}+\text { H.c. }\right) \text {, }
$$

where $\left(n_{j}, \alpha_{j}\right)$ denotes the indices of the unit cell $n_{j}$ and the particular resonator $\alpha_{j}$ that the $j$ th emitter is coupled to. Summing up, the global generic Hamiltonian that we will consider contains the sum of the three terms,

$$
H=H_{S}+H_{B}+H_{\text {int }}
$$

\section{PHOTON-MEDIATED INTERACTIONS}

In order to obtain the photon-mediated interactions emerging in this general class of models defined by $H$, we will assume to be in the Born-Markov regime in which the photonic bath timescales are much faster than the induced emitter ones. This allows us to adiabatically eliminate the photons [49,50], resulting in photon-mediated interactions containing both a real and an imaginary part,

$$
J_{i j}-i \gamma_{i j}=\sum_{E} \frac{\left\langle 0\left|\sigma_{j} H_{\mathrm{int}}\right| E\right\rangle\left\langle E\left|H_{\mathrm{int}} \sigma_{i}^{\dagger}\right| 0\right\rangle}{\Delta+i 0^{+}-E},
$$

which leads to unitary and nonunitary emitter dynamics, respectively. Here, $|E\rangle$ is an eigenstate of the free part of the Hamiltonian [Eq. (5) with $H_{\text {int }}=0$ ], $E$ is its energy, and $|0\rangle$ is the global vacuum state of the system $\hat{a}_{k, \alpha}|0\rangle=\sigma_{j}|0\rangle=0$ for all $k, \alpha$, and $j$.

In this manuscript, we are interested only in the situations where $\gamma_{i j} \equiv 0$, which can be obtained within this approximation assuming that $\Delta$ lies in a band-gap region of the model, i.e., $\Delta \notin \omega_{\alpha}(k)$ for any $\alpha$ or $k$. Note that this regime can always be obtained in these models since we are considering finite bath hoppings, which impose a finite bandwidth for the energy bands of the model $\omega_{\alpha}(k)$. By tuning the emitters into those frequency regions, the photon-mediated interactions result in an effective spin model,

$$
H_{\mathrm{eff}}=\sum_{i, j}\left(J_{i j} \sigma_{i}^{\dagger} \sigma_{j}+\text { H.c. }\right)
$$


where $J_{i j}$ can be written as (see the Appendices)

$$
J_{i j}=\frac{|g|^{2}}{2 \pi} \int_{-\pi}^{\pi} d k\left(\Delta \mathbb{I}-h_{B}(k)\right)_{\alpha_{i} \alpha_{j}}^{-1} e^{i k n_{j i}},
$$

with $n_{j i} \equiv n_{j}-n_{i}$ being the interemitter distance and $\mathbb{I}$ the identity matrix. The integrand can be expanded as

$$
\begin{aligned}
{\left[\left(\Delta+i 0^{+}\right) \mathbb{I}-h_{B}(k)\right]_{\alpha_{i} \alpha_{j}}^{-1}=} & \frac{1}{\operatorname{det}\left[\Delta \mathbb{I}-h_{B}(k)\right]} \\
& \times \operatorname{adj}\left[\Delta \mathbb{I}-h_{B}(k)\right]_{\alpha_{i} \alpha_{j}},
\end{aligned}
$$

where $\operatorname{adj}\left[\Delta \mathbb{I}-h_{B}(k)\right]$ is the adjugate matrix, which is the transpose of the cofactor matrix, and which turns out to be built with the minors of $\Delta \mathbb{I}-h_{B}(k)$. Both the numerator and the denominator of (9) are $O\left(N_{c}\right)$ th-degree polynomials of the matrix elements of $h_{B}(k), \delta_{\alpha}(k)$, and $f_{\alpha \beta}(k)$ [Eq. (2)]. As we are assuming that the hopping terms in the photonic bath are local up to a finite number of neighbors, both $\delta_{\alpha}(k)$ and $f_{\alpha \beta}(k)$ can be written as finite sums of powers of $e^{ \pm i k}$. This implies that the integrand of $J_{i j}$ is, up to a factor $e^{i k n_{j i}}$, a quotient of $O\left(N_{c}\right)$ th-degree polynomials of $e^{i k}$. Taking the change of variable $y=e^{i k \operatorname{sgn}\left(n_{j i}\right)}, J_{i j}$ transforms into

$$
J_{i j}=\frac{|g|^{2}}{2 \pi i} \oint d y y^{\left|n_{j i l}\right|} \frac{A_{\alpha_{i} \alpha_{j}}(y)}{B_{\alpha_{i} \alpha_{j}}(y)} .
$$

As a consequence of the previous discussion, both $A_{\alpha_{i} \alpha_{j}}(y)$ and $B_{\alpha_{i} \alpha_{j}}(y)$ are polynomials in $y$ with a finite degree $O\left(N_{c}\right)$. As the poles of the integrand are inside or outside the unit circle, but never in the circle (see Appendix C), we can apply the residue's theorem, taking into account just the poles inside the unit circle, $\left\{y_{l}^{<}\right\}$,

$$
J_{i j}=|g|^{2} \sum_{l} \operatorname{Res}\left[\frac{A_{\alpha_{i} \alpha_{j}}(y) y^{\left|n_{j i}\right|}}{B_{\alpha_{i} \alpha_{j}}(y)}, y=y_{l}^{<}\right] .
$$

Since $B_{\alpha_{i} \alpha_{j}}(y)$ is a finite-order polynomial (see previous discussion), the degree of the poles $\left\{y_{p}^{<}\right\}$is also finite. Using the definition of residue, each term in (11) will be proportional to $\left(y_{p}^{<}\right)^{\left|n_{j i}\right|}$. This means that irrespective of the model considered, $J_{i j}$ is a finite sum of decaying exponentials (notice that $\left|y_{l}^{<}\right|<$ $1)$, such that it can be written

$$
J_{i j}=\sum_{l} C_{l} e^{-\left|n_{j i}\right| / \xi_{l}},
$$

where the parameters $\left\{C_{l}, \xi_{l}\right\}$ depend on the particular model considered. Note that this formally discards the possibility of having purely power-law interactions mediated by onedimensional photonic models. In the next section, however, we will see how one can still mimic power-law interactions within certain distance regimes by breaking some of the assumptions of the generic model that we have considered.

\section{EMULATING POWER-LAW INTERACTIONS}

As mentioned in Sec. I, long-range coherent interactions, understood as decaying with a power-law behavior, can lead to qualitatively different phenomena in interacting spin models as compared to short-range ones. For example, these longrange models are known to break conventional Lieb-Robinson bounds on the spread of correlations [11-14,18,51], lead to exotic many-body phases $[8,10,18,19,23,24]$, modify the area law [15,22], or yield fast equilibration times [16], among other phenomena. For this reason, it is still a relevant question to see, in spite of the results that we derived in the previous section, whether it is still possible to obtain such powerlaw behavior by breaking some of the assumptions of the considered generic model.

In this section, we briefly sketch two possibilities: in Sec. IV A, we show how one can approximate power-law decay within a distance window by summing a finite set of exponentials in a controlled fashion by going beyond local light-matter couplings. Then, in Sec. IV B, we study baths with power-law hoppings and show that they give rise to nonanalytical energy dispersions, which allow one to obtain power-law asymptotic decays for the interactions (with the same decay exponent of the original hopping model).

\section{A. Mimicking power laws by adding finite number of exponentials}

The fact that power laws can be built by summing up exponentials is well known and used in many other fields (e.g., in economy [52]). In quantum optical setups, this has already been exploited, e.g., in Ref. [30], to obtain approximate power laws by the use of Raman-assisted transitions. As we showed in the previous section, in multipartite lattices, such finite sums of exponentials appear in a natural way [see Eq. (12)] and can lead to power-law behavior in certain distance windows. For example, taking a tripartite lattice described by a general Hamiltonian [see Fig. 2(a)],

$$
h_{B}(k)=\left(\begin{array}{ccc}
-2 t_{1} \cos k & t_{12} & t_{13} \\
t_{12} & -2 t_{2} \cos k & t_{23} \\
t_{13} & t_{23} & -2 t_{3} \cos k
\end{array}\right) \text {, }
$$

where $t_{n}$ is the nearest-neighbor hopping between the resonators in different sublattices, and $t_{\alpha \beta}$ describes the coupling between the $\alpha$ th and $\beta$ th resonator within the unit cells, we can find that $J_{i j}$ displays a power-law behavior in certain regimes. To illustrate it, we plot in Fig. 2(b) the effective emitter interactions $J_{i j}$ for a given set of parameters (see caption) that we found mimic a power-law behavior for distances up to $\sim 10$ neighbors. The different colors represent different values of the emitter's energy $\Delta$, which lead to different approximate power-law exponents. This is shown more explicitly in Fig. 2(c), where we plot the results of the fitting $J_{i j}$ to a power law for short distances. These interactions would already allow one to probe long-range interacting phenomena for small emitter distances such as done with trapped ions, e.g., in Refs. [10,51], where effective power-law behaviors were also obtained for chains of around 10 ions. However, in order to fully explore the unconventional phenomena appearing in long-range interacting models, it would be desirable to extend its range to longer distances (ideally to the thermodynamic limit).

One way of extending the range of the power-law interactions would be to use the methods of optimal exponential expansions of power-law decays (see Ref. [52]). These methods provide a recipe to obtain the optimal $\left(C_{l}, \xi_{l}\right)$ parameters one needs to input in Eq. (12) to approximate a power-law decay 

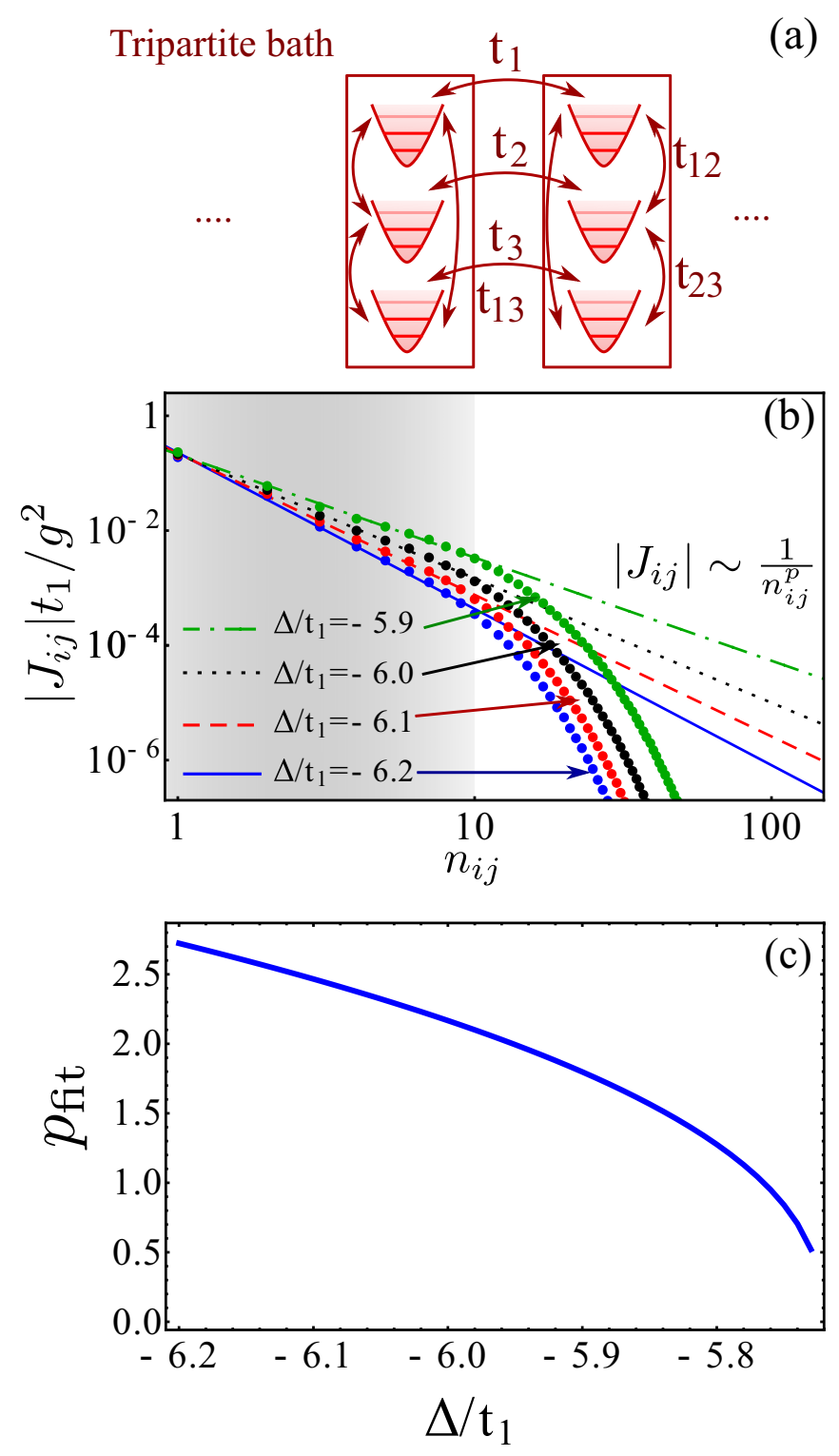

FIG. 2. (a) General scheme of a tripartite lattice with hoppings defined in the Hamiltonian of Eq. (13). (b) Absolute value of $J_{i j}$ as a function of the emitter's distance $n_{i j}$ for two emitters coupled to sublattice $\alpha=1$. The points are the exact values and the lines are the fits to a power law $\sim n_{i j}^{-p}$ for $1 \leqslant n_{i j} \leqslant 10$. We take $t_{1}$ as the unit of energy. The couplings are $t_{2}=2.3 t_{1}, t_{3}=2.8 t_{1}$, and $t_{12}=t_{23}=t_{13}=$ $0.3 t_{1}$. The values of $\Delta$ are in the legends. As shown there, $J_{\text {eff }}$ follows a power law up to $n_{i j} \simeq 10$ (shaded area). (c) Approximate powerlaw exponent as a function of $\Delta$ for the values of the parameters of (b). The exponent is obtained by a linear fit for small distances, obtaining values $p_{\text {fit }} \in(0.5,2.5)$.

with exponent $v$ up to a given distance, i.e.,

$$
\begin{aligned}
C_{\alpha, \mathrm{opt}} & =\left(\frac{e}{\beta^{\alpha}}\right)^{v}, \\
\xi_{\alpha, \mathrm{opt}} & =\frac{\beta^{\alpha}}{v}
\end{aligned}
$$
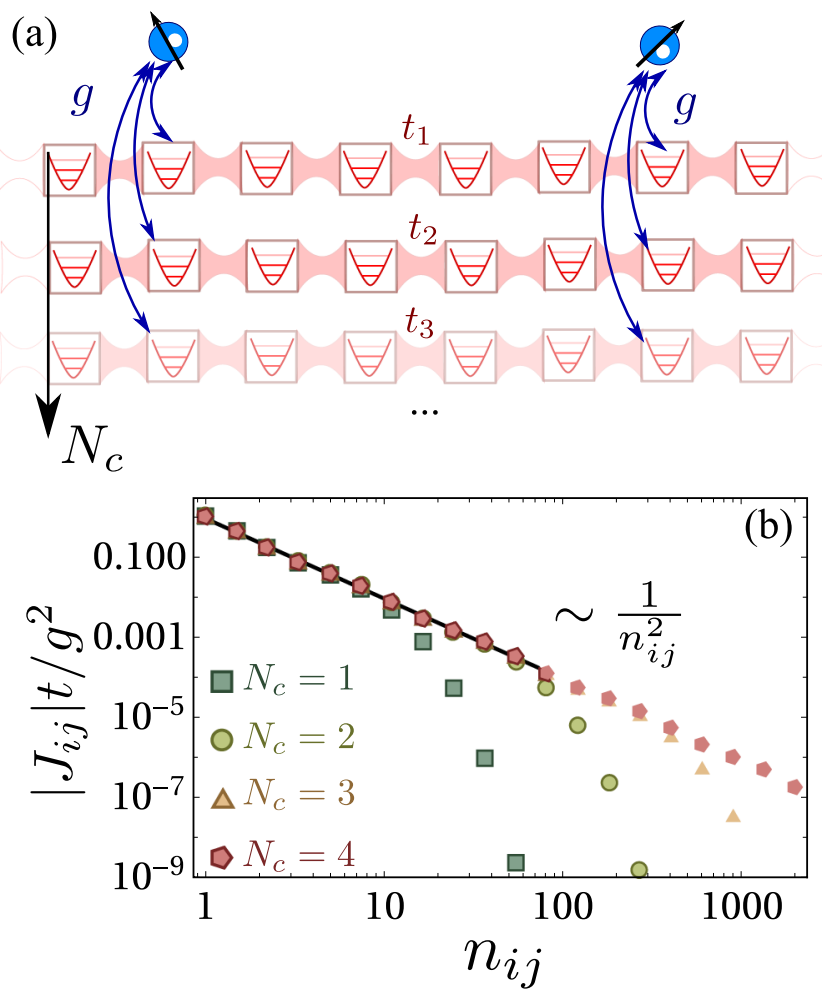

FIG. 3. (a) Scheme of the nonlocal coupling of emitters to several uncoupled wave guides. (b) Result of applying the optimal expansion method of Ref. [52] to obtain $J_{i j} \approx 1 / n_{i j}^{2}$ interactions. In solid black, we plot the exact $1 / n_{i j}^{2}$, while the markers are the results of approximation with $N_{c}=1,2,3,4$ wave guides. The wave-guide parameters have to be engineered such that the weights and decay length match those of Eqs. (14) and (15), using $\beta=6$.

where $\beta$ is a number that must be optimized to match the power-law behavior. Unfortunately, the relation between the physical parameters of generic multipartite lattices and the resulting parameters $\left(C_{l}, \xi_{l}\right)$ in Eq. (12) is difficult to unravel, precluding their application.

There is, however, one way in which the application of these optimal expansion methods would be conceptually straightforward (although experimentally challenging). It requires allowing for nonlocal light-matter couplings, that is, that the emitters couple to several resonators $a_{n, \alpha=1, \ldots, N_{c}}$ simultaneously. These couplings have recently been achieved with giant atoms in circuit QED platforms [46,47]. The idea consists in defining a multipartite lattice with $N_{c}$ resonators per unit cell, but where they only interact with the resonators of different unit cells, that is, the bath consists of a set of uncoupled one-dimensional wave guides with energy dispersions $\omega_{\alpha}(k)=\tilde{\omega}_{\alpha}-2 t_{\alpha} \cos (k)$, where we explicitly write the possibility that the resonators of each uncoupled wave guide may have different energy $\tilde{\omega}_{\alpha}$. If the $j$ th emitter couples nonlocally to all the resonators in a given unit cell $n_{j}$ [see Fig. 3(a)], the photon-mediated interactions will be given by the addition of the photon-mediated interactions of each uncoupled wave guide because $h_{B}(k)$ is diagonal (see Appendix D). Thus, $J_{i j}$ can be written as in Eq. (12), but 
(a)
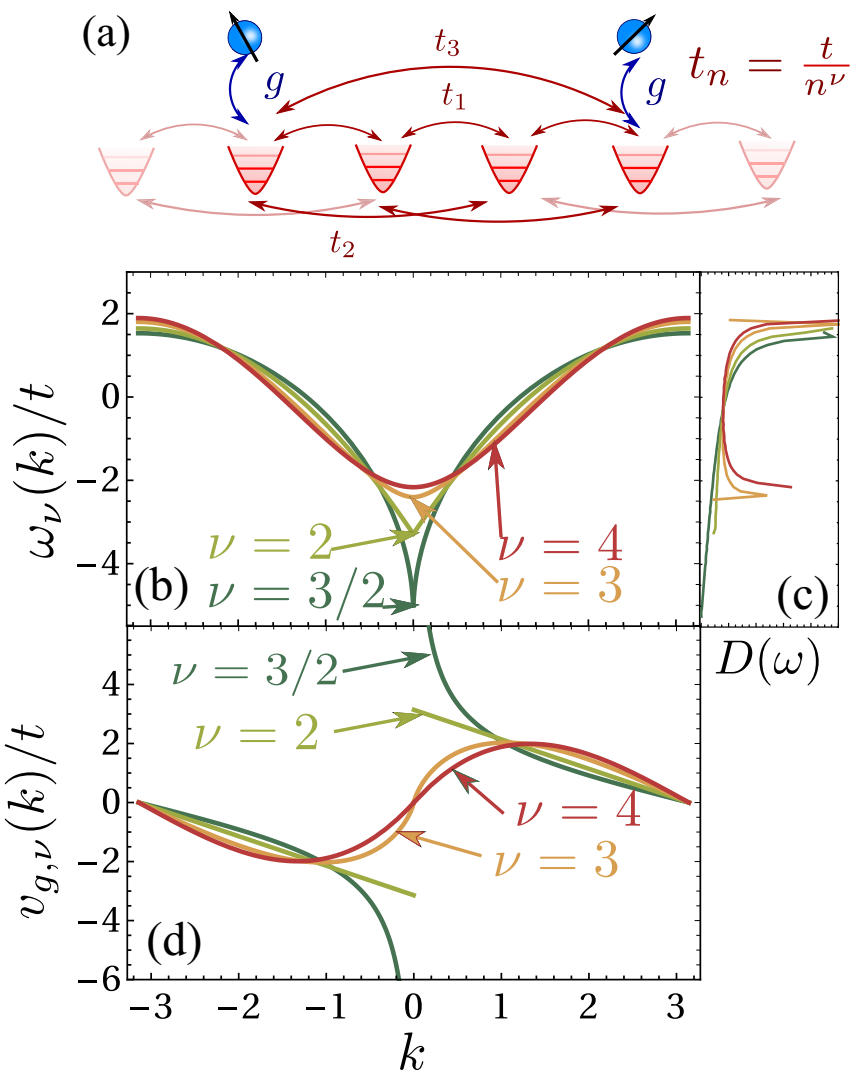

FIG. 4. (a) Scheme of emitters coupled to a one-dimensional model with long-range hoppings $t_{n}=-\frac{t}{n^{v}}$ between $n$ neighbors. (b) Bath energy dispersion $\omega_{\nu}(k) / t$, (c) density of states, and (d) group velocity $v_{g, v}(k) / t$ for several power-law exponents $v=$ $3 / 2,2,3,4$, as depicted in the legend.

where $\left(C_{l}, \xi_{l}\right)$ are given by (see Refs. [53,54])

$$
\begin{gathered}
C_{\alpha} \approx-\frac{g^{2} \xi_{\alpha}}{2 t_{\alpha}}, \\
\xi_{\alpha} \approx \sqrt{\frac{t_{\alpha}}{D_{\alpha}}},
\end{gathered}
$$

where $D_{\alpha}=\Delta-\tilde{\omega}_{\alpha}-2 t_{\alpha}$ is the effective detuning of the emitter's energy to the lower band edge [we are assuming the emitter's frequency lies below the band edge of all $\left.\omega_{\alpha}(k)\right]$. This means that by tuning $\left(t_{\alpha}, D_{\alpha}\right)$, one can independently tune the weight and range of the exponentials and match it to those of Eqs. (14) and (15). In Fig. 3, we illustrate this procedure by plotting how one can obtain a $J_{i j} \sim 1 / n_{i j}^{2}$ interaction for increasing distance ranges up to 1000 lattice sites by coupling the emitter nonlocally to an increasing number of wave guides.

\section{B. Long-range hopping models}

The other possibility that goes beyond the initial bath assumptions of Sec. III consists in allowing for longer-range bath hoppings scaling as $t_{n}=t / n^{\nu}$, such as depicted in Fig. 4(a). These long-range hopping models appear naturally in trapped ion systems (see, e.g., Ref. [20]) with power-law exponent $v=3$. Free-space photons also mediate power-law dipole-dipole couplings between neutral atoms $[1,2]$ with a combination of exponents $v=1,2,3$. In the deeply subwavelength regime (near field), where $v=3$ dominates, the dissipative terms are smaller than the dipole-dipole couplings and can therefore be used to explore these long-range hopping models. Such power-law hoppings also appear in magnonic networks where superconducting loops can be used to enhance the range of the interactions to obtain $v<3$, as recently proposed in Ref. [55]. In the spirit of the manuscript of providing results that are as general as possible, we consider the situation where the exponent can have any value (limited by some physical bounds that we discuss afterwards).

The bath Hamiltonian of such photonic long-range hopping models can be described by a simple Bravais lattice with $N_{c}=$ 1 , and its energy dispersion depends explicitly on the powerlaw exponent $v$ :

$$
\omega_{\nu}(k)=-t\left[\operatorname{Li}_{\nu}\left(e^{i k}\right)+\operatorname{Li}_{\nu}\left(e^{-i k}\right)\right] .
$$

Here, $\operatorname{Li}_{v}(z)$ is the polylogarithm function of the order of $v$. These energy dispersions are finite for every $k$ as long as $v>1$, since $\omega_{1}(k)$ displays a logarithmic singularity around $k=0$. Thus, we will restrict our discussion to models with $v>1$. As an illustration, in Figs. 4(b) and 4(c), we plot the energy dispersion and associated density of states $D(\omega)$ for models with power-law exponents $v=3 / 2,2,3$, and 4 . As expected for large exponents, both the energy dispersion and density of states tend to converge to the nearest-neighbor case of $\omega(k) \approx-2 t \cos (k)$, with two van Hove singularities at the band edges (see, e.g., $[53,54,56])$. When the exponent decreases, however, the longer-range hoppings strongly modify the band structure and associated density states. In particular, we observe that $\omega_{v}(k)$ features a visible nonanalytical kink around $k \approx 0$, which is more evident when we calculate the group velocity of the model, i.e., $v_{g, v}(k)=\partial_{k} \omega_{\mu}(k)$, which we plot in Fig. 4(d) for the same power-law exponents. There, we observe, for example, the finite discontinuous jump of the group velocity for $v=2$, i.e., $v_{g, 2}(k) \approx \pi \operatorname{sign}(k)-k$. The jump becomes bigger as $v \rightarrow 1$, finally showing a $1 / k$ divergence when $v=1$. This increase of the group velocity around $k=0$ leads to strong modification of the density of states, e.g., canceling the lower edge Van Hove singularity as $v \rightarrow 1$.

Like in other structured baths [57], such nonanalytical behavior of the density of states will result in non-Markovian quantum dynamics when the emitter's frequencies are tuned with the nonanalytical regions. In this manuscript, however, we will focus only on characterizing the effective emitter's interactions $J_{i j}$ in the regime where one can still adiabatically eliminate the photonic bath (Born-Markov regime) by assuming $\Delta$ lies far enough from the band edges $[53,54,56]$. Since this bath can be written as a simple Bravais lattice, the expression of Eq. (8) simplifies to

$$
J_{i j}^{\nu}=\frac{|g|^{2}}{2 \pi} \int_{-\pi}^{\pi} d k \frac{e^{i k n_{j i}}}{\Delta-\omega_{\nu}(k)} .
$$

Differently from the baths we have considered up to now, the $\omega_{\nu}(k)$ energy dispersion has a branch cut that is along the imaginary axis that will lead to qualitatively different behavior (see Appendix E for a complete discussion of the integration). To characterize $J_{i j}^{v}$ for all distance regimes, it is convenient to 
distinguish the situations when the emitter's energy lies in the upper or lower band-gap region.

(i) Upper band-gap regime. This corresponds to situations when $\Delta>\omega_{v}(\pi)$. In that case, the photon-mediated interactions can always be written as a sum of two contributions,

$$
J_{i j}^{v}=J_{i j, \text { pole }}^{v}+J_{i j, \mathrm{BC}}^{v},
$$

which can be obtained by defining a closed contour in the complex plane that includes the line integral of Eq. (19). Then, $J_{\text {pole }}^{v}$ is the contribution of the complex poles of the denominator of the integrand of Eq. (19) embedded by the chosen closed contour (see Appendix E for details). Their contribution can be obtained using the Residue theorem by expanding $\omega_{\nu}(k)$ close to the position where the pole is expected, i.e., $\omega_{\nu}( \pm \pi+i y) / t \approx \omega_{\nu}(\pi) / t+A_{\nu} y^{2}$, for $y \ll 1$. Using that expansion, we find

$$
J_{i j, \text { pole }}^{v} \approx(-1)^{\left|n_{i j}\right|} \frac{|g|^{2} \xi_{v}}{2 t A_{v}} e^{-\left|n_{i j}\right| / \xi_{v}},
$$

where $\xi_{v}=\sqrt{A_{v} t / D_{u}}, D_{u}=\Delta-\omega_{v}(\pi)$, and $A_{v}$ is a numerical constant that depends on the exponent $\nu$. The other contribution to $J_{i j}^{v}$ comes from the detour we have to take when using the Residue theorem to avoid the branch cut of the integrand. This contribution can be written as

$$
\begin{aligned}
J_{i j, \mathrm{BC}}^{v}= & \int_{0}^{\infty} G_{\nu}(y) e^{-y\left|n_{i j}\right|} \approx-\frac{|g|^{2}}{\pi} \int_{0}^{\infty} d y \\
& \times \frac{\operatorname{Im}\left[\omega_{v}(\varepsilon+i y)\right] e^{-y\left|n_{i j}\right|}}{\left\{\Delta-\operatorname{Re}\left[\omega_{\nu}(\varepsilon+i y)\right]\right\}^{2}+\operatorname{Im}\left[\omega_{v}(\varepsilon+i y)\right]^{2}} .
\end{aligned}
$$

Irrespective of $\Delta$, this term will eventually dominate the long-distance behavior of $J_{i j}^{v}$ since the pole contribution is exponentially attenuated. Due to the exponential term $e^{-y\left|n_{i j}\right|}$ of the integrand of Eq. (22), the long-distance behavior of $J_{i j, \mathrm{BC}}^{v}$ will be dominated by the behavior of $G_{v}(y)$ when $y \ll 1$, which we find to be $G_{v}(y \ll 1) \propto y^{\nu-1}$. Using that $\int_{0}^{\infty} y^{\alpha} e^{-y d}=\Gamma(1+\alpha) / d^{1+\alpha}$, we can show then that the photon-mediated interactions scale as

$$
J_{i j, \mathrm{BC}}^{v} \propto-\frac{|g|^{2} t}{D_{u}^{2}} \frac{1}{\left|n_{i j}\right|^{v}},
$$

in the asymptotic limit $\left(\left|n_{i j}\right| / \xi_{v} \gg 1\right)$. Thus, the dipole-dipole interactions $J_{i j}^{v}$ inherit the power-law exponent from the hopping model. This behavior is illustrated in Fig. 5(a), where we plot the result of numerically integrating $J_{i j}^{v}$ as a function of the emitter's distance $n_{i j}$ for the same power-law exponents chosen for Fig. 4, and for a detuning $D_{u}=0.05 t$. There, we clearly observe that after an initial exponential decay of the interactions coming from the pole contribution, $J_{i j}^{v}$ features an asymptotic power-law scaling (dashed line) with the same power-law exponent as the original bath model. The crossover between the exponential and power-law behavior occurs at distances $\left|n_{i j}\right| \sim \xi_{v}$, that is, when the pole contribution starts to be strongly attenuated. Note that this regime was already explored for $v=3$ in the context of trapped ions [20], obtaining similar results.

(ii) Lower band-gap regime. This regime corresponds to situations when $\Delta<\omega_{\nu}(0)$, and remarkably it leads to different phenomena. The main difference with respect to the upper band-gap situation is that the pole contribution can be shown

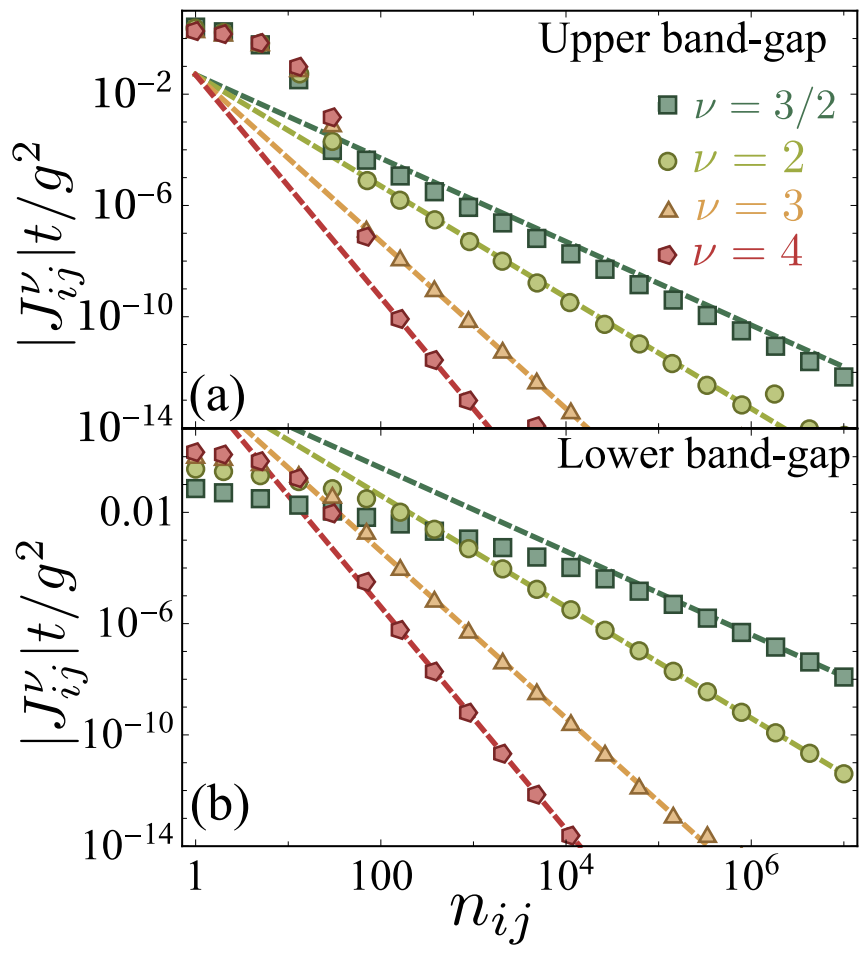

FIG. 5. Effective emitter's interaction $J_{i j}^{v}$ induced by the photonic long-range hopping models as a function of the emitter's distance $n_{i j}$ for $v=1.5,2,3$, and 4 (see legend). The markers are the result of the numerical integration of Eq. (19), whereas the dashed lines are the asymptotic scaling laws that match those of the original hopping model. We fix the detuning between the emitter's energy and the bottom and top of the band to $0.05 \mathrm{~J}: \Delta=\omega_{v}(0 / \pi) \pm 0.05 \mathrm{~J}$ in (a) and (b), respectively.

to be strictly zero, $J_{\text {pole }}^{v} \equiv 0$, such that $J_{i j}^{\nu}$ comes solely from the branch-cut detour contribution $J_{i j, \mathrm{BC}}^{v}$ (see the Appendices). This makes its short-distance behavior strongly dependent on the particular $v$ exponent of the hopping model and qualitatively very different from the upper band-gap situation. This is clearly seen in Fig. 5(b), where we plot the $J_{i j}^{v}$ for the same detunings and exponent as in Fig. 5(a), but for the lower band gap. There, we observe the following:

(a) For $v \leqslant 3$, the $J_{i j}^{v}$ does not display the initial exponential decay coming from the pole contribution. In fact, for the case of $v=2$, we can find that the initial decay follows a logarithmic law,

$$
J_{i j}^{\nu=2} \approx \frac{|g|^{2}\left\{\gamma+\ln \left[\left|n_{i j}\right| D_{l} /(\pi t)\right]\right\}}{\pi^{2} t},
$$

for distances $\left|n_{i j}\right| \ll \pi t / D_{l}$. For $v=3$, we also derived a (more cumbersome) analytical expression (see the Appendices) which shows a similar logarithmic decay.

(b) For $v>3$, even though $J_{i j}^{v}$ comes entirely from the branch-cut contribution of Eq. (22), it starts displaying an approximated exponential decay [see Fig. 5(b)]. The mathematical reason is that one can find an approximated pole of Eq. (19), whose residue can be approximated by

$$
\frac{J_{i j, \mathrm{pole}, *}^{v} t}{|g|^{2}} \approx \frac{\xi_{v}^{*}}{2 C_{v}} e^{-\left|n_{i j}\right| / \xi_{v}^{*}},
$$


with $\xi_{v}^{*}=\sqrt{\left|C_{\nu}\right| t / D_{l}}$ with $D_{l}=\omega_{\nu}(0)-\Delta>0$, and $C_{\nu}$ coming from the expansion $\omega_{\nu}\left(0^{+}+i y\right) / t \approx \omega_{v}(0) / t+C_{v} y^{2}$ when $y \ll 1$. When $v \gg 1, C_{v} \approx-1$, recovering the results of the nearest-neighbor model that were given in Eqs. (16) and (17). Note that this is expected since when $v \gg 1$, the longerrange hoppings are negligible with respect to the nearestneighbor ones.

\section{CONCLUSIONS}

Summing up, we have derived several general results for the limits of (coherent) photon-mediated interactions induced by one-dimensional photonic environments. First, we have shown that under the standard assumptions of locality of light-matter (rotating-wave) couplings and photon hoppings, the photon-mediated interactions can always be written as a finite sum of exponentials. Thus, they can only display power-law behavior in small-distance windows. In addition, we have proposed two ways of extending the range of such power-law behavior by considering models that go beyond the previous assumptions. For example, by nonlocally coupling the qubits to several one-dimensional wave guides, one could considerably extend the range of the power-law behavior of the interactions in a controlled fashion. Finally, we have also considered the photon-mediated interactions appearing in one-dimensional baths with power-law hoppings. These models display nonanalytical energy dispersions, which lead to interactions that inherit the asymptotic scaling of the original hopping model. In addition, we find situations where the photon-mediated interactions are not exponentially attenuated in any distance window. We foresee that other baths with similar nonanalytical energy dispersions, such as resonator arrays coupled with $X_{i} X_{j}$ couplings or critical spin baths, will also display similar power-law asymptotic scalings. Another interesting direction to explore is whether these conclusions hold as well for models in the ultrastrong-coupling regime, where such photon-mediated interactions have recently started being explored [58].

\section{ACKNOWLEDGMENTS}

We acknowledge Ignacio Cirac, Johannes Knörzer, Daniel Malz, Martí Perarnau, and Cosimo C. Rusconi for inspiring and fruitful discussions. E.S.-B. acknowledges ERC Advanced Grant QUENOCOBA under the EU Horizon 2020 program (Grant Agreement No. 742102). A.G.-T. acknowledges funding from Project No. PGC2018-094792B-I00 (MCIU/AEI/FEDER, UE), CSIC Research Platform PTI-001, and CAM/FEDER Project No. S2018/TCS-4342 (QUITEMAD-CM).

\section{APPENDIX A: INTRODUCTION}

In these appendices, we provide more details on the general diagonal form of the bath Hamiltonian (Appendix B), the derivation of the general photon-mediated interactions $J_{i j}$ (Appendix C), the generalization of $J_{i j}$ to the case in which the light-matter couplings are not fully local (Appendix D), and, finally, a detailed analysis of the photon-mediated interactions for the long-range hopping model (Appendix E).

\section{APPENDIX B: DIAGONAL FORM OF $H_{B}$}

The bosonic Hamiltonian $H_{B}$ of Eq. (3) can be diagonalized. As $h_{B}(k)$ is Hermitian,

$$
h_{B}(k)=P(k) D(k) P^{\dagger}(k),
$$

where $P(k)$ is unitary and

$$
D(k)=\operatorname{diag}\left[\omega_{1}(k), \omega_{2}(k), \ldots, \omega_{N_{c}}(k)\right],
$$

with $\left\{\omega_{n}(k)\right\}_{n=1}^{N_{c}}$ being the eigenvalues of $h_{B}(k)$. Then, defining a new set of bosonic operators $\left\{\hat{\alpha}_{n, k}\right\}_{n=1}^{N_{c}}$,

$$
\left(\begin{array}{c}
\hat{\alpha}_{1, k} \\
\vdots \\
\hat{\alpha}_{N_{c}, k}
\end{array}\right) \equiv P^{\dagger}(k)\left(\begin{array}{c}
\hat{a}_{1, k} \\
\vdots \\
\hat{a}_{N_{c}, k}
\end{array}\right) .
$$

$H_{B}$ then reads

$$
H_{B}=\sum_{k} \sum_{n=1}^{N_{c}} \omega_{n}(k) \hat{\alpha}_{n, k}^{\dagger} \hat{\alpha}_{n, k}
$$

\section{APPENDIX C: EFFECTIVE PHOTON-MEDIATED INTERACTIONS}

In order to derive Eq. (8) from Eq. (6), we write the bosonic operators $a_{n_{j}, \alpha}$ of $H_{\text {int }}$ in momentum space and in terms of the $\alpha$ modes of Eq. (B3),

$$
\begin{aligned}
a_{n_{j}, \alpha} & =\frac{1}{\sqrt{N}} \sum_{k} e^{i k n_{j}} \hat{a}_{k, \alpha} \\
& =\frac{1}{\sqrt{N}} \sum_{k} e^{i k n_{j}} \sum_{\beta=1}^{N_{c}} P_{\alpha_{j} \beta}^{*}(k) \hat{\alpha}_{k, \beta} .
\end{aligned}
$$

From this and Eq. (4), the only eigenvalues contributing to the sum in (6) are $|E\rangle=\hat{\alpha}_{k, n}^{\dagger}|0\rangle$, with $E=\omega_{n}(k)$. After taking the thermodynamic limit $N \rightarrow \infty$ in Eq. (6),

$$
J_{i j}=\frac{|g|^{2}}{2 \pi} \int_{-\pi}^{\pi} d k \sum_{\beta=1}^{N_{c}} \frac{P_{\alpha_{i} \beta}(k) P_{\alpha_{j} \beta}^{*}(k)}{\Delta+i 0^{+}-\omega_{\beta}(k)} e^{i k n_{j i}} .
$$

The sum in the integrand can be rewritten as

$$
\sum_{\beta, \gamma=1}^{N_{c}} P_{\alpha_{i} \beta}(k) \frac{\delta_{\beta \gamma}}{\Delta+i 0^{+}-\omega_{\beta}(k)}\left(P^{\dagger}(k)\right)_{\gamma \alpha_{j}} .
$$

Notice that $P(k)$ is the matrix which diagonalizes $h_{B}(k)$ [Eq. (B1)]. Using the well-known property

$$
\begin{gathered}
P(k) D(k) P^{\dagger}(k)=h_{B}(k) \\
\Rightarrow P(k) f(D(k)) P^{\dagger}(k)=f\left(h_{B}(k)\right),
\end{gathered}
$$

we see that $(\mathrm{C} 3)$ is $\left[\left(\Delta+i 0^{+}\right) \mathbb{I}-h_{B}(k)\right]_{\alpha_{i} \alpha_{j}}^{-1}$. Introducing this in (C2), we get (8).

In addition, notice that the inverse $\left[\left(\Delta+i 0^{+}\right) \mathbb{I}-h_{B}(k)\right]^{-1}$ always exists. To prove this, we write down the determinant of $\left(\Delta+i 0^{+}\right) \mathbb{I}-h_{B}(k)$,

$$
\operatorname{det}\left[\left(\Delta+i 0^{+}\right) \mathbb{I}-h_{B}(k)\right]=\prod_{\beta=1}^{N_{c}}\left[\Delta+i 0^{+}-\omega_{\beta}(k)\right] .
$$


As $\Delta$ is not embedded in the bands, i.e., $\Delta \neq \omega_{\alpha}(k) \forall k, \alpha$, this determinant is different from 0 . In consequence, its inverse exists.

A corollary of this last result is that the integrand of $J_{i j}$ [Eq. (10)] has no poles in the unit circle, $\left|y_{l}\right| \neq 1$. This is due to the fact that the polynomial of the denominator of the integrand of $J_{i j}$ is proportional to the determinant of $\left[\Delta+i 0^{+}-\right.$ $\left.h_{B}(k)\right]^{-1}$ [see Eqs. (8)-(10)]. We just proved that provided $\Delta$ is not in the bands of the model, this determinant is different from $0 \forall k \in \mathbb{R}$, that is, for $|y|=1$. In consequence, all the poles are inside or outside the circle, but never in the circle.

\section{APPENDIX D: BEYOND POINTLIKE LIGHT-MATTER COUPLINGS}

Let us assume that each emitter couples to a finite number of contiguous sites,

$$
H_{\mathrm{int}}=\sum_{j=1}^{2} \sigma_{j}^{+} \sum_{\alpha, n} g_{\alpha, n}^{j} a_{n, \alpha}+\text { H.c. }
$$

Introducing this interaction Hamiltonian in the definition of $J_{i j}$, given by Eq. (6), and taking the thermodynamic limit,

$$
\begin{aligned}
J_{i j}= & \frac{1}{2 \pi} \sum_{\alpha_{i} \alpha_{j} n_{i} n_{j}}\left(g_{\alpha_{i} n_{i}}^{i}\right)^{*} g_{\alpha_{j} n_{j}}^{j} \\
& \times \int d k e^{i k\left(n_{j}-n_{i}\right)}\left[\left(\Delta+i 0^{+}\right) \mathbb{I}-h_{B}(k)\right]_{\alpha_{i} \alpha_{j}}^{-1} .
\end{aligned}
$$

The integrand is identical to the pointlike case considered in the main text [Eq. (8)], being the only difference is the sum over the couplings to the different sites and sublattices. Therefore, $J_{i j}$ is again a sum of exponentials of $\left|n_{j i}\right|$, each weighted with the couplings $\left(g_{\alpha_{i} n_{i}}^{i}\right)^{*} g_{\alpha_{j} n_{j}}^{j}$.

As a corollary of this, we can derive the effective interaction in the particular case in which there is no interaction in each sublattice, so $f_{\alpha \beta}(k)=0$ and $\omega_{\alpha}(k)=\delta_{\alpha}(k)$ [see Eq. (2)], and the $i$ th qubit is coupled to the position $n_{i}$ of each sublattice with the same coupling strength $g$. Considering this in Eq. (D2), we get

$$
J_{i j}=\frac{|g|^{2}}{2 \pi} \sum_{\alpha} \int_{-\pi}^{\pi} d k \frac{e^{i n_{j i}}}{\Delta-\omega_{\alpha}(k)},
$$

that is, the effective photon-mediated interactions are the sum of the ones independently induced by each energy band $\omega_{\alpha}(k)$.

\section{APPENDIX E: ASYMPTOTIC SCALING OF THE INTERACTIONS IN LONG-RANGE PHOTONIC MODELS}

As we have shown in the main text, the energy dispersion of the photonic bath model with power-law hoppings of exponent $v$ is given by

$$
\omega_{\nu}(k)=-t\left[\operatorname{Li}_{v}\left(e^{i k}\right)+\operatorname{Li}_{v}\left(e^{-i k}\right)\right],
$$

where $\operatorname{Li}(y)$ is the polylogarithm function. This function is defined by a power series,

$$
\operatorname{Li}_{v}(z)=\sum_{k=1}^{\infty} \frac{z^{k}}{k^{v}}
$$

valid for any complex $v$ and all complex arguments within $|z|<1$, although it can be analytically continued to the whole complex plane. Assuming a local coupling to the bath, with coupling strength $g$, the photon-mediated interactions are given by

$$
\frac{J_{i j}^{v}}{|g|^{2}}=\frac{1}{2 \pi} \int_{-\pi}^{\pi} d k \frac{e^{i k\left|n_{i j}\right|}}{\Delta-\omega_{\nu}(k)} .
$$

In the case of finite-range hopping models, this integral was calculated by making the change of variable $z=e^{i k}$ and was shown to be solely given the contribution of the poles within the unit circle. Here, instead, the contour cannot be simply closed because $\omega_{v}(k)$ has a branch cut along the imaginary axis of $\operatorname{Im} k \in(-\infty, \infty)$ inherited by the branch cut of the polylogarithm function. As a consequence, $J_{i j}^{v}$ will contain additional contributions coming from the detours to avoid the branch cut. Let us now provide approximated expressions of $J_{i j}^{v}$ for both the situation where the emitters' energy lies above and below the band, which we will show to lead to qualitatively different behavior.

Upper band gap. Let us start with the case where $\Delta>$ $\omega_{v}(k)$ for all $k$, and denote as $D_{u}$ the energy difference between the emitter's energy and the upper band edge, i.e., $D_{u}=\Delta-\omega_{v}( \pm \pi)>0$. Taking $k$ as a complex variable, we can find that the denominator of the integrand of Eq. (E3) has four complex poles close to $\pm \pi$, but slightly above or below the real axis. To find an approximated expression for the poles, we can expand $\omega_{\nu}(k)$ close to that point, and finding it can always be written as

$$
\omega_{\nu}( \pm \pi+i y) / t \approx \omega_{\nu}( \pm \pi) / t+A_{\nu} y^{2}
$$

for $|y| \ll 1$, and where the higher-order terms of the expansion remain real. This means that to the lowest order of $y$, the poles can be approximated by

$$
\begin{gathered}
k_{\pi, \pm} \approx \pi \pm i \sqrt{D_{u} / A_{v}}, \\
k_{-\pi, \pm} \approx-\pi \pm i \sqrt{D_{u} / A_{\nu}} .
\end{gathered}
$$

Thus, if we define the contour as depicted with dashed arrows in Fig. 6(a), the integral of Eq. (E3) can be shown to be given by two contributions: the one of the poles embedded within the contour, plus the ones of the two detours along the branch cut (red arrows),

$$
J_{i j}^{v}=J_{i j, \text { pole }}^{v}+J_{i j, \mathrm{BC}}^{v} .
$$

The only poles that contribute are the ones with the positive imaginary part, i.e., $k_{ \pm \pi,+}$, whose contribution can be obtained by calculating their associated residue. This can be done by writing $\Delta-\omega_{v}(k) \approx A_{v}\left(k-k_{ \pm \pi,+}\right)\left(k-k_{ \pm \pi,-}\right)$ for the $k$ 's close to the poles, which yields

$$
\frac{J_{i j, \mathrm{pole}}^{v} t}{g^{2}} \approx \frac{(-1)^{\left|n_{i j}\right|}}{2} \frac{\xi_{v}}{A_{v}} e^{-\left|n_{i j}\right| / \xi_{v}}
$$

where $\xi_{u}=\sqrt{A_{v} t / D_{u}}$ provides the localization length of the interaction and its strength. Note that because the poles lie along the integration contour, they contribute with half of its residue. This can be proven more rigorously by making the integration along this line and making use of the identity 
(a)
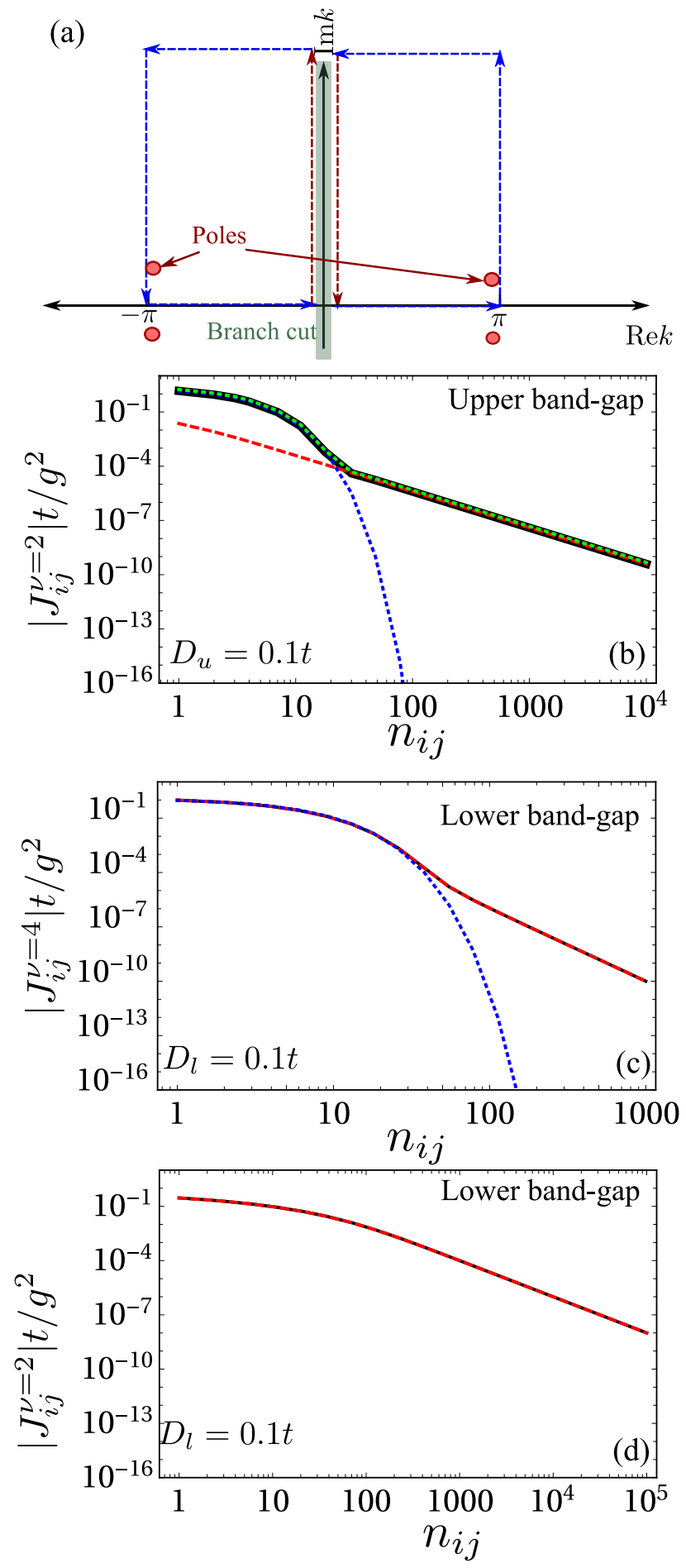

FIG. 6. (a) Contour of integration (dashed blue line) to obtain $J_{i j}^{\nu}$ as an approximated expression of Eq. (E3). (b) $J_{i j}^{\nu=2}$ as a function of the distance $n_{i j}$ for an emitter tuned to the upper band gap. We also separately plot the contribution of the poles (dotted blue line) and the branch-cut detour one (dashed red line). The dotted green line shows the sum of the two contributions given by Eqs. (E8) and (E9), respectively. (c) $J_{i j}^{v=4}$ calculated from numerically integrating Eq. (E3) for an emitter tuned to the lower band gap. The dotted blue line shows the approximated expression found in Eq. (E15). (d) $J_{i j}^{\nu=2}$ calculated from numerically integrating Eq. (E3) for an emitter tuned to the lower band gap. The dashed red line shows the analytical expression obtained in Eq. (E16). $\frac{1}{x \pm i 0^{+}}=\mp i \pi \delta(x)+P(1 / x)$, or by shifting the domain of the integration of Eq. (E3) to $(0,2 \pi)$, as done in Ref. [20].

The branch-cut detour contribution can be written in the following compact expression:

$$
\begin{aligned}
\frac{J_{\mathrm{BC}}^{\nu} t}{|g|^{2}} \approx & -\frac{1}{\pi} \int_{0}^{\infty} d y \\
& \times \frac{\operatorname{Im}\left[\omega_{\nu}(\varepsilon+i y)\right] e^{-y\left|n_{i j}\right|}}{\left\{\Delta-\operatorname{Re}\left[\omega_{\nu}(\varepsilon+i y)\right]\right\}^{2}+\operatorname{Im}\left[\omega_{\nu}(\varepsilon+i y)\right]^{2}} \\
= & \int_{0}^{\infty} G_{\nu}(y) e^{-y\left|n_{i j}\right|} .
\end{aligned}
$$

Since the pole contribution is exponentially damped for larger distances, the asymptotic scaling of $J_{i j}^{v}$ will always be provided by $J_{\mathrm{BC}}^{\nu}$. Due to the $e^{-y\left|n_{i j}\right|}$ dependence of the integrand, the $J_{\mathrm{BC}}^{v}$ behavior at long distances is dominated by the dependence of $G_{v}(y)$ for $y \ll 1$, which we find to be $G_{v}(y) \approx B_{v} y^{\nu-1}$, with $B_{v}$ being a constant that depends on $\Delta$ and the exponent $v$. Since $\int_{0}^{\infty} y^{\alpha} e^{-y d}=\Gamma(1+\alpha) / d^{1+\alpha}$ for $\alpha>-1, d>0$, and $\Gamma(x)$ is the $\Gamma$ function, the final asymptotic scaling of the photon-mediated interactions can be shown to inherit the same power-law behavior of the hopping model, i.e., $J_{i j}^{v} \propto 1 /\left|n_{i j}\right|^{v}$ for $n_{i j} / \xi_{v} \gg 1$.

As an illustration that the expressions derived above reproduce well the behavior of $J_{i j}^{v}$, in Fig. 6(b) we plot together the $J_{i j}^{v}$ for $v=2$ obtained from the direct numerical integration of Eq. (E3) (solid black line), and its different contributions: the pole contribution given by Eq. (E8) (dotted blue line), the branch-cut contribution as defined in Eq. (E9) (dashed red line), and the sum of the two (dotted green line). The other power-law exponents lead to qualitatively similar phenomena.

Lower band gap. When the emitter's energy lies in the lower band gap, that is, $\Delta<\omega_{\nu}(k)$, the denominator has no pole, such that $J_{i j}^{v}$ is only given by the branch-cut contribution, i.e., $J^{v}=J_{\mathrm{BC}}^{v}$. That is why, in these regions, one should try to find the poles close to zero momentum, e.g., $k=0^{ \pm}+i y$. However, when expanding $\omega_{\nu}(k)$ around it, we find that differently from $\omega_{\nu}( \pm \pi+i y)$, it contains both real and imaginary terms, such that no solution can be found. In particular, we find (for integer $v$ )

$$
\begin{gathered}
\operatorname{Re} \omega_{\nu}\left(0^{+}+i y\right) \approx \omega_{\nu}(0)+C_{\nu} y^{2}, \\
\operatorname{Im} \omega_{\nu}\left(0^{+}+i y\right) \approx D_{\nu} y^{\nu-1},
\end{gathered}
$$

with $D_{v}>0$ for all $v$, but where $C_{v}$ behaves differently depending on $v$,

$$
\begin{gathered}
C_{2}=\frac{1}{2}, \\
C_{3}=\left[\ln (y)-\frac{3}{2}\right], \\
C_{v>3}<0 .
\end{gathered}
$$

The different behavior of such expansions for the different exponents will lead to qualitatively different photon-mediated interactions $J_{i j}^{v}$. Thus, it is convenient to separately analyze the different situations that can appear:

(a) When $v>3$, the $J_{i j}^{v}$ for short distances starts to features an exponential decay like in the upper band-gap situation. This is illustrated in Fig. 6(c) for $v=4$. Note that this behavior 
is expected because, when $v \gg 1$, one should recover the limit of nearest-neighbor hoppings which solely feature an exponential decay [Eqs. (16) and (17)]. This can be reconciled mathematically by noticing that when $v>3$, the imaginary contribution of $\omega_{v}\left(0^{+}+i y\right)$ starts to be subleading compared to the real part [see Eqs. (E10) and (E11)]. In that case, if one neglects this imaginary part, the integrand of $J_{i j}^{v}$ in Eq. (E3) will have a pole, whose contribution can be approximated by

$$
\frac{J_{i j, \mathrm{pole}, *}^{v} t}{|g|^{2}} \approx \frac{\xi_{v}^{*}}{2 C_{v}} e^{-\left|n_{i j}\right| / \xi_{v}^{*}}
$$

with $\xi_{v}^{*}=\sqrt{-C_{v} t / D_{l}}$, where $D_{l}=\omega_{v}(0)-\Delta>0$ [remember that $C_{v>3}<0$; see Eq. (E14)]. For $v \gg 1, C_{v} \approx-1$, recovering the results of the nearest-neighbor model that were given in Eqs. (16) and (17).

(b) When $v<3$, on the contrary, the imaginary part of $\omega_{\nu}\left(0^{+}+i y\right)$ is of higher order in $y$ than the real one [see Eqs. (E10) and (E11)], and the initial exponential decay is not present. This is illustrated in Fig. 6(d), where we calculate numerically $J_{i j}^{\nu=2}$ (solid black line), and compare it with an analytical expression (dashed red line) that can be obtained in that case from the expansion of $\omega_{\nu=2}\left(0^{+}+i y\right)$. This analytical expression reads

$$
J_{i j}^{\nu=2}=-\frac{|g|^{2}\left\{-\cos \left(x_{i j}\right) \operatorname{ci}\left(x_{i j}\right)+\sin \left(x_{i j}\right)\left[\pi-2 \operatorname{si}\left(x_{i j}\right)\right]\right\}}{2 \pi^{2} t},
$$

where $x_{i j}=\left|n_{i j}\right| D_{l} /(\pi t)$, and $\operatorname{ci}(x)$ and $\operatorname{si}(x)$ are the cosine and sine integral functions [59]. When $x_{i j} \ll 1$,

$$
J_{i j}^{\nu=2} \approx \frac{|g|^{2}\left[\gamma+\ln \left(x_{i j}\right)\right]}{\pi^{2} t},
$$

with $\gamma$ being the Euler constant. This is a very interesting regime because it leads to one-dimensional photon-mediated interactions with no exponential attenuation, and with a range larger than the original photonic model.

(c) The case of $v=3$ is more difficult to treat analytically due to the logarithmic divergence that one finds in $C_{3}$ [see Eq. (E13)]. In order to find an approximated expression, let us first note that one can approximate $G_{3}(y)$ by the lowest-order expansion of $\omega_{v=3}\left(0^{+}+i y\right)$, which reads

$$
G_{3}(y) \approx-\frac{y^{2}}{2\left(\left\{D_{l}^{2}+y^{2}[\ln (y)-3 / 2]\right\}^{2}+\pi^{2} y^{4} / 2\right)}
$$

By a numerical study, we showed that this approximation is already enough to reproduce well the behavior for most distances. Then, it is convenient to divide the integrand of $J_{i j, \mathrm{BC}}^{v=3}$ into two functions,

$$
\frac{J_{i j, \mathrm{BC}}^{\nu=3}}{g^{2}}=\int_{0}^{\infty} d y G_{3}(y) e^{-y\left|n_{i j}\right|} \approx \int_{0}^{\infty} d y F(y) H\left(y,\left|n_{i j}\right|\right),
$$

where

$$
\begin{gathered}
F(y)=-\frac{1}{2\left(\left\{D_{l}^{2}+y^{2}[\ln (y)-3 / 2]\right\}^{2}+\pi^{2} y^{4} / 2\right)}, \\
H(y, d)=y^{2} e^{-y d} .
\end{gathered}
$$

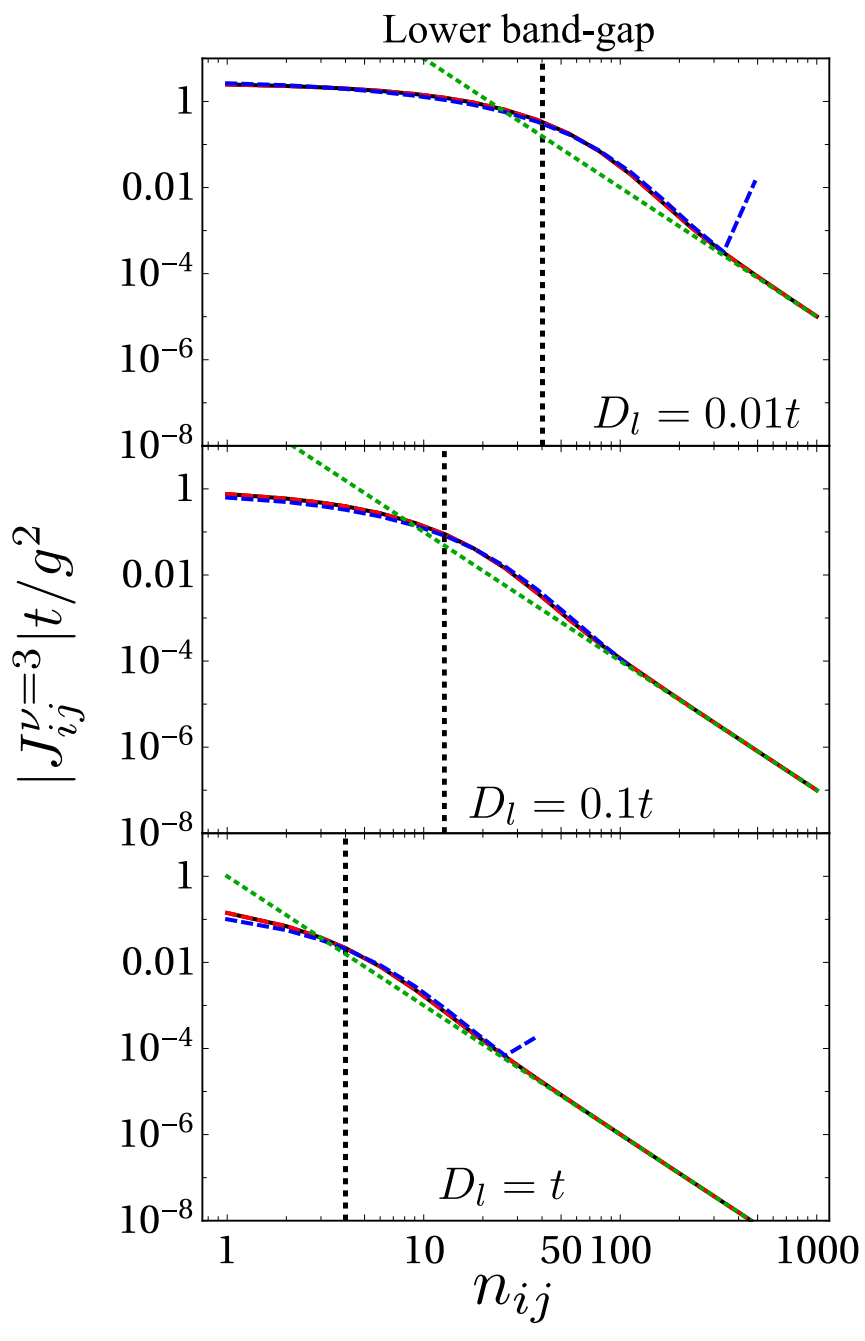

FIG. 7. $\left|J_{i j}^{\nu=3}\right|$ calculated from numerically integrating Eq. (E3) for an emitter tuned to the lower band gap (solid black line). The dashed red line shows the result of approximating the integrand of the branch-cut contribution as in Eq. (E18). The dashed green line shows the asymptotic expression of Eq. (E26). The dotted blue line shows the approximated expression we found in Eq. (E31). The three panels correspond to increasing values of $D_{l} / t$ (see legend), whereas the vertical dashed line corresponds to the critical distance $4 / \sqrt{D_{l} / t}$ where the transition between the short- and long-range behavior is expected.

The function of $H(y, d)$ scales as

$$
\begin{gathered}
H(y \ll 1, d) \approx y^{2}, \\
H(y \gg 1, d) \approx y^{2} e^{-y d},
\end{gathered}
$$

and has a maximum $y_{h} \approx 2 / d$. The function $F(y)$ scales as

$$
\begin{gathered}
F(y \ll 1) \approx-\frac{1}{2 \delta^{2}}, \\
F(y \gg 1) \approx-\frac{1}{2 y^{4} \ln (2 / d)^{2}},
\end{gathered}
$$

and has a maximum $y_{f} \approx \sqrt{D_{l} / t} / 2$. Thus, it will be the ratio $y_{f} / y_{h}$ that will determine a transition between two qualitatively different regimes. For example, when $\left|n_{i j}\right| \gg 4 / \sqrt{D_{l} / t}$, 
one can find the same asymptotic scaling as in the upper band-gap regime,

$$
\frac{J_{i j, \mathrm{BC}}^{\nu=3} t}{g^{2}} \approx-\frac{t^{2}}{\left|n_{i j}\right|^{3} D_{l}^{2}} .
$$

To find an expression for $J_{i j, \mathrm{BC}}^{\nu=3}$ in the short-distance regime, $\left|n_{i j}\right| \ll 4 / \sqrt{D_{l} / t}$, we found it is a good approximation to replace $\ln (y) \rightarrow \ln (2 / d)$, which is justified due to the peaked behavior of $H(y, d)$ around $y_{h}=2 / d$. Like this, one can rewrite $F(y)$ as the sum of

$$
\begin{aligned}
F(y)= & -\frac{|A|^{2}}{4 D_{l}^{2} y^{2}}\left[\left(\frac{1}{y^{2}-A}+\text { c.c. }\right)\right. \\
& \left.+\frac{\operatorname{Re} A}{i \operatorname{Im} A}\left(\frac{1}{y^{2}-A}-\text { c.c. }\right)\right],
\end{aligned}
$$

where

$$
A=\frac{2 i D_{l}}{\pi+i[3-2 \ln (2 / d)]} .
$$

The advantage of this rewriting is that now the integral has a closed analytical expression in terms of cosine and sine integrals,

$$
\int_{0}^{\infty} d y \frac{e^{-y d}}{y^{2}-A}=d q(-\sqrt{A} d)
$$

with $q(x)$,

$$
q(x)=\frac{2 \operatorname{ci}(x) \sin (x)+\cos (x)[\pi-2 \operatorname{si}(x)]}{2 x} .
$$

Thus,

$$
\begin{aligned}
\frac{J_{i j, \mathrm{BC}}^{\nu=3} t}{g^{2}} \approx & -\frac{|A|^{2} d}{2 D_{l}^{2}}\left\{\operatorname{Re}\left[q\left(-\sqrt{A}\left|n_{i j}\right|\right)\right]\right. \\
& \left.+\frac{\operatorname{Re} A}{\operatorname{Im} A} \operatorname{Im}\left[q\left(-\sqrt{A}\left|n_{i j}\right|\right)\right]\right\}
\end{aligned}
$$

In Fig. 7, we illustrate the behavior of all these approximations. In the different panels, we compare the result of numerically integrating Eq. (E3) for $v=3$ and several detunings $D_{l} / t=0.01,0.1,1$ (solid black line) with the different approximated expressions that we found. For example, the dashed red line shows the result of calculating the branchcut detour contribution approximating the integrand by the expression given by Eq. (E18). The dashed green line shows the asymptotic contribution found in Eq. (E26), whereas the dotted blue line shows the expression we found for short distances in Eq. (E31). We note that at long distances, it starts to deviate significantly due to the approximation $\ln (y) \rightarrow$ $\ln (2 / d)$ we perform to be able to obtain an analytical expression. However, this occurs when the asymptotic expression of Eq. (E26) already captures the results well.
[1] R. H. Lehmberg, Phys. Rev. A 2, 883 (1970).

[2] R. H. Lehmberg, Phys. Rev. A 2, 889 (1970).

[3] M. Saffman, T. G. Walker, and K. Mølmer, Rev. Mod. Phys. 82, 2313 (2010).

[4] K. Hammerer, A. S. Sørensen, and E. S. Polzik, Rev. Mod. Phys. 82, 1041 (2010).

[5] D. Porras and J. I. Cirac, Phys. Rev. Lett. 92, 207901 (2004).

[6] K. Kim, M.-S. Chang, S. Korenblit, R. Islam, E. E. Edwards, J. K. Freericks, G.-D. Lin, L.-M. Duan, and C. Monroe, Nature (London) 465, 590 (2010).

[7] P. Hauke, F. M. Cucchietti, A. Müller-Hermes, M.-C. Bañuls, J. I. Cirac, and M. Lewenstein, New J. Phys. 12, 113037 (2010).

[8] A. W. Sandvik, Phys. Rev. Lett. 104, 137204 (2010).

[9] M. Maik, P. Hauke, O. Dutta, J. Zakrzewski, and M. Lewenstein, New J. Phys. 14, 113006 (2012).

[10] R. Islam, C. Senko, W. Campbell, S. Korenblit, J. Smith, A. Lee, E. Edwards, C.-C. Wang, J. Freericks, and C. Monroe, Science 340, 583 (2013).

[11] P. Hauke and L. Tagliacozzo, Phys. Rev. Lett. 111, 207202 (2013).

[12] J. Jünemann, A. Cadarso, D. Pérez-García, A. Bermudez, and J. J. García-Ripoll, Phys. Rev. Lett. 111, 230404 (2013).

[13] Z.-X. Gong, M. Foss-Feig, S. Michalakis, and A. V. Gorshkov, Phys. Rev. Lett. 113, 030602 (2014).

[14] M. Foss-Feig, Z.-X. Gong, C. W. Clark, and A. V. Gorshkov, Phys. Rev. Lett. 114, 157201 (2015).

[15] T. Koffel, M. Lewenstein, and L. Tagliacozzo, Phys. Rev. Lett. 109, 267203 (2012).

[16] M. Kastner, Phys. Rev. Lett. 106, 130601 (2011).
[17] D. Vodola, L. Lepori, E. Ercolessi, A. V. Gorshkov, and G. Pupillo, Phys. Rev. Lett. 113, 156402 (2014).

[18] Z.-X. Gong, M. F. Maghrebi, A. Hu, M. L. Wall, M. Foss-Feig, and A. V. Gorshkov, Phys. Rev. B 93, 041102(R) (2016).

[19] Z.-X. Gong, M. F. Maghrebi, A. Hu, M. Foss-Feig, P. Richerme, C. Monroe, and A. V. Gorshkov, Phys. Rev. B 93, 205115 (2016).

[20] P. Nevado and D. Porras, Phys. Rev. A 93, 013625 (2016).

[21] Z. Eldredge, Z.-X. Gong, J. T. Young, A. H. Moosavian, M. Foss-Feig, and A. V. Gorshkov, Phys. Rev. Lett. 119, 170503 (2017).

[22] Z.-X. Gong, M. Foss-Feig, F. G. S. L. Brandão, and A. V. Gorshkov, Phys. Rev. Lett. 119, 050501 (2017).

[23] M. F. Maghrebi, Z.-X. Gong, and A. V. Gorshkov, Phys. Rev. Lett. 119, 023001 (2017).

[24] B. Žunkovič, M. Heyl, M. Knap, and A. Silva, Phys. Rev. Lett. 120, 130601 (2018).

[25] E. M. Purcell, H. C. Torrey, and R. V. Pound, Phys. Rev. 69, 37 (1946).

[26] J. D. Joannopoulos, P. R. Villeneuve, and S. Fan, Nature (London) 386, 143 (1997).

[27] V. P. Bykov, Sov. J. Quantum Electron. 4, 861 (1975).

[28] G. Kurizki, Phys. Rev. A 42, 2915 (1990).

[29] S. John and J. Wang, Phys. Rev. Lett. 64, 2418 (1990).

[30] J. S. Douglas, H. Habibian, C.-L. Hung, A. Gorshkov, H. J. Kimble, and D. E. Chang, Nat. Photon. 9, 326 (2015).

[31] A. González-Tudela, C.-L. Hung, D. E. Chang, J. I. Cirac, and H. Kimble, Nat. Photon. 9, 320 (2015).

[32] A. González-Tudela and J. I. Cirac, Phys. Rev. A 97, 043831 (2018). 
[33] J. Perczel and M. D. Lukin, Phys. Rev. A 101, 033822 (2020).

[34] A. González-Tudela and J. I. Cirac, Quantum 2, 97 (2018).

[35] I. García-Elcano, A. González-Tudela, and J. Bravo-Abad, arXiv:1903.07513.

[36] L. Ying, M. Zhou, M. Mattei, B. Liu, P. Campagnola, R. H. Goldsmith, and Z. Yu, Phys. Rev. Lett. 123, 173901 (2019).

[37] E. Sánchez-Burillo, C. Wan, D. Zueco, and A. GonzálezTudela, Phys. Rev. Research 2, 023003 (2020).

[38] A. Goban, C.-L. Hung, S.-P. Yu, J. Hood, J. Muniz, J. Lee, M. Martin, A. McClung, K. Choi, D. Chang, O. Painter, and H. Kimblemblrm, Nat. Commun. 5, 3808 (2014).

[39] P. Lodahl, S. Mahmoodian, and S. Stobbe, Rev. Mod. Phys. 87, 347 (2015).

[40] Y. Liu and A. A. Houck, Nat. Phys. 13, 48 (2017).

[41] M. Mirhosseini, E. Kim, V. S. Ferreira, M. Kalaee, A. Sipahigil, A. J. Keller, and O. Painter, Nat. Commun. 9, 3706 (2018).

[42] J. Rui, D. Wei, A. Rubio-Abadal, S. Hollerith, J. Zeiher, D. M. Stamper-Kurn, C. Gross, and I. Bloch, arXiv:2001.00795.

[43] S. J. Masson and A. Asenjo-Garcia, arXiv:1912.06234.

[44] I. de Vega, D. Porras, and J. Ignacio Cirac, Phys. Rev. Lett. 101, 260404 (2008).

[45] L. Krinner, M. Stewart, A. Pazmino, J. Kwon, and D. Schneble, Nature (London) 559, 589 (2018).

[46] A. F. Kockum, A. Miranowicz, S. De Liberato, S. Savasta, and F. Nori, Nat. Rev. Phys. 1, 19 (2019).

[47] B. Kannan, M. Ruckriegel, D. Campbell, A. F. Kockum, J. Braumüller, D. Kim, M. Kjaergaard, P. Krantz, A. Melville, B. M. Niedzielski et al., arXiv:1912.12233.
[48] Note that by taking this definition, we are assuming that the unit of distance will be given by the lattice constant. Thus, from now on, all the lengths (and momenta) will be units of the lattice constant (or its inverse).

[49] C. Cohen-Tannoudji, J. Dupont-Roc, G. Grynberg, and P. Thickstun, Atom-photon Interactions: Basic Processes and Applications (Wiley Online Library, New York, 1992).

[50] G. W. Gardiner and P. Zoller, Quantum Noise, 2nd ed. (Springer-Verlag, Berlin, 2000).

[51] P. Richerme, Z.-X. Gong, A. Lee, C. Senko, J. Smith, M. FossFeig, S. Michalakis, A. V. Gorshkov, and C. Monroe, Nature (London) 511, 198 (2014).

[52] T. Bochud and D. Challet, arXiv:physics/0605149v2.

[53] T. Shi, Y.-H. Wu, A. González-Tudela, and J. I. Cirac, New J. Phys. 20, 105005 (2018).

[54] G. Calajó, F. Ciccarello, D. Chang, and P. Rabl, Phys. Rev. A 93, 033833 (2016).

[55] C. C. Rusconi, M. J. A. Schuetz, J. Gieseler, M. D. Lukin, and O. Romero-Isart, Phys. Rev. A 100, 022343 (2019).

[56] T. Shi, Y.-H. Wu, A. González-Tudela, and J. I. Cirac, Phys. Rev. X 6, 021027 (2016).

[57] S. John and T. Quang, Phys. Rev. A 50, 1764 (1994).

[58] J. Román-Roche, E. Sánchez-Burillo, and D. Zueco, arXiv:2001.07643.

[59] M. Abramowitz, I. A. Stegun et al., Handbook of Mathematical Functions, Applied Mathematics Series, No. 55 (National Bureau of Standards, U.S. Department of Commerce, 1964). 